\title{
CORRIGENDA
}

\section{Early, sustained efficacy of adeno-associated virus vector-mediated gene therapy in glycogen storage disease type la}

DD Koeberl, BD Sun, TV Damodaran, T Brown, DS Millington, DK Benjamin Jr, A Bird, A Schneider,

S Hillman, M Jackson, RM Beaty and YT Chen

Gene Therapy (2007) 14, 281. doi:10.1038/sj.gt.3302903

Correction to: Gene Therapy (2006) 13, 1281-1289. The acknowledgment sentence is shown below:

doi:10.1038/sj.gt.3302774

G6Pase-KO mice were provided by Dr Janice Chou at the

Since the above publication, the authors have realised that they did not acknowledge Dr Janice Chou in the Acknowledgments section.

National Institutes of Health.

The authors would like to apologise for this error.

\section{Gene delivery to human sweat glands: a model for cystic fibrosis gene therapy}

\author{
H Lee ${ }^{1,2}$, DR Koehler ${ }^{1,3}$, CY Pang ${ }^{1,4,5}$, RH Levine ${ }^{5}, \mathrm{P} \mathrm{Ng}^{6}$, DJ Palmer ${ }^{6}$, PM Quinton ${ }^{7}$ and J Hu ${ }^{1,2,3}$ \\ ${ }^{1}$ Research Institute, The Hospital for Sick Children, Toronto, Canada; ${ }^{2}$ Institute of Medical Science, University of Toronto, Toronto, \\ Canada; ${ }^{3}$ Department of Laboratory Medicine and Pathology, University of Toronto, Toronto, Canada; ${ }^{4}$ Department of Physiology, \\ University of Toronto, Toronto, Canada; ${ }^{5}$ Department of Surgery, University of Toronto, Toronto, Canada; ${ }^{6}$ Department of Molecular and \\ Human Genetics, Baylor College of Medicine, Houston, TX, USA and ${ }^{7}$ Department of Pediatrics, UCSD School of Medicine University \\ of California, San Diego, La Jolla, CA, USA
}

Gene Therapy (2007) 14, 281. doi:10.1038/sj.gt.3302905

Correction to: Gene Therapy (2005) 12, 1752-1760. doi:10.1038/sj.gt.3302587

Since the publication of the above paper, the authors have noticed an omission in affiliation 7 for Dr Quinton. The correct affiliation is shown below:
${ }^{7}$ Department of Pediatrics, UCSD School of Medicine University of California, San Diego, La Jolla, CA, USA; Division of Biomedical Sciences, University of California, Riverside, Riverside, CA, USA.

The authors would like to apologise for this error. 